\title{
Microvascular endothelial dysfunction is associated with albuminuria
}

Citation for published version (APA):

Martens, R. J. H., Houben, A. J. H. M., Kooman, J. P., Berendschot, T. T. J. M., Dagnelie, P. C., van der Kallen, C. J. H., Kroon, A. A., Leunissen, K. M. L., van der Sande, F. M., Schaper, N. C., Schouten, J. S. A. G., Schram, M. T., Sep, S. J. S., Sörensen, B. M., Henry, R. M. A., \& Stehouwer, C. D. A. (2018). Microvascular endothelial dysfunction is associated with albuminuria: the Maastricht Study. Journal of Hypertension, 36(5), 1178-1187. https://doi.org/10.1097/HJH.0000000000001674

Document status and date:

Published: 01/05/2018

DOI:

10.1097/HJH.0000000000001674

Document Version:

Publisher's PDF, also known as Version of record

Document license:

Taverne

Please check the document version of this publication:

- A submitted manuscript is the version of the article upon submission and before peer-review. There can be important differences between the submitted version and the official published version of record.

People interested in the research are advised to contact the author for the final version of the publication, or visit the DOI to the publisher's website.

- The final author version and the galley proof are versions of the publication after peer review.

- The final published version features the final layout of the paper including the volume, issue and page numbers.

Link to publication

\footnotetext{
General rights rights.

- You may freely distribute the URL identifying the publication in the public portal. please follow below link for the End User Agreement:

www.umlib.nl/taverne-license

Take down policy

If you believe that this document breaches copyright please contact us at:

repository@maastrichtuniversity.nl

providing details and we will investigate your claim.
}

Copyright and moral rights for the publications made accessible in the public portal are retained by the authors and/or other copyright owners and it is a condition of accessing publications that users recognise and abide by the legal requirements associated with these

- Users may download and print one copy of any publication from the public portal for the purpose of private study or research.

- You may not further distribute the material or use it for any profit-making activity or commercial gain

If the publication is distributed under the terms of Article $25 \mathrm{fa}$ of the Dutch Copyright Act, indicated by the "Taverne" license above, 


\title{
Original Article
}

\section{Microvascular endothelial dysfunction is associated with albuminuria: the Maastricht Study}

\author{
Remy J.H. Martens ${ }^{a, b}$, Alfons J.H.M. Houben ${ }^{c, d}$, Jeroen P. Kooman ${ }^{a, b}$, Tos T.J.M. Berendschot ${ }^{e}$, \\ Pieter C. Dagnelie ${ }^{\text {d,f,g }}$, Carla J.H. van der Kallen ${ }^{c, d}$, Abraham A. Kroon ${ }^{c, d}$, Karel M.L. Leunissen ${ }^{a, b}$, \\ Frank M. van der Sande ${ }^{a}$, Nicolaas C. Schaper ${ }^{c, d, f}{ }^{,}$, Jan S.A.G. Schouten ${ }^{e}$, Miranda T. Schram ${ }^{c, d, h}$, \\ Simone J.S. Sep ${ }^{c, d}$, Ben M. Sörensen ${ }^{c, d}$, Ronald M.A. Henry ${ }^{c, d, h}$, and Coen D.A. Stehouwer ${ }^{c, d}$
}

See editorial comment on page 1036

Objective: Albuminuria is thought to be a biomarker of microvascular and macrovascular endothelial dysfunction. However, direct evidence for an association of microvascular endothelial dysfunction with albuminuria is limited. In addition, experimental data suggest a stronger association of microvascular endothelial dysfunction with albuminuria in individuals with than in those without diabetes.

Methods: We examined cross-sectional associations of flicker light-induced retinal arteriolar dilation $(n=2095)$ and heat-induced skin hyperemia $(n=1508)$ with $24-h$ albuminuria in the population-based, diabetes-enriched Maastricht Study. We used linear regression analyses to adjust for age, sex, type 2 diabetes, and cardiovascular disease risk factors. In addition, we tested for statistical interaction with type 2 diabetes.

Results: Median [interquartile range] albuminuria was 6.5 [3.9-11.6] $\mathrm{mg} / 24 \mathrm{~h}$ and $8.2 \%$ had albuminuria at least $30 \mathrm{mg} / 24 \mathrm{~h}$. After adjustment, albuminuria was 1.168 (95\% confidence interval, 1.046-1.303) times greater in participants in the quartile with the smallest flicker lightinduced retinal arteriolar dilation relative to those with the greatest dilation, and this association was stronger in participants with type 2 diabetes $\left(P_{\text {interaction }}<0.10\right)$. Further, each 100 percentage points lower heat-induced skin hyperemia was associated with a 1.022 (1.010-1.035) times greater albuminuria in participants with type 2 diabetes, whereas it was not associated with albuminuria in nondiabetic participants $\left(P_{\text {interaction }}<0.10\right)$.

Conclusion: This is the first population-based study that provides direct evidence that microvascular endothelial dysfunction is associated with albuminuria, and that this association is stronger in individuals with than in those without type 2 diabetes.

Keywords: albuminuria, cardiovascular disease, diabetes mellitus, endothelial dysfunction, endothelium, microalbuminuria, microcirculation
Abbreviations: CKD-EPI, Chronic Kidney Disease Epidemiology Collaboration; CVD, cardiovascular disease; DVA, dynamic vessel analyzer; eGFR, estimated glomerular filtration rate; $\mathrm{HbA} 1 \mathrm{c}$, hemoglobin $\mathrm{A} 1 \mathrm{c}$; $\mathrm{NO}$, nitric oxide; $\mathrm{Q}$, quartile; UAE, urinary albumin excretion; VEGF, vascular endothelial growth factor

\section{INTRODUGTION}

1buminuria is a marker of risk of not only renal 1 failure and cardiovascular disease (CVD) [1], but 1 also of retinopathy [2], heart failure [3], impaired cognitive performance [4], and depression [5]. A leading hypothesis to explain these associations is that albuminuria is a biomarker of generalized (i.e. microvascular and macrovascular) endothelial dysfunction [2,5-8].

This concept posits that endothelial dysfunction of renal arterioles and capillaries (i.e. the renal microcirculation) increases intraglomerular pressure and glomerular capillary wall permeability, and thereby causes albuminuria [6]. Concomitantly, endothelial dysfunction of the extrarenal microcirculation contributes to retinopathy, cognitive decline, depression, and heart failure with preserved ejection fraction $[2,5,7,8]$, whilst endothelial dysfunction in coronary and carotid arteries (i.e. the macrocirculation) leads to atherothrombotic CVD [6].

Indeed, there is strong evidence for the presence of endothelial dysfunction in the macrocirculation of individuals with albuminuria [9-11].

In contrast, evidence for endothelial dysfunction in the microcirculation of individuals with albuminuria is primarily indirect. It derives mainly from studies using plasma biomarkers [12-14] and the transcapillary escape rate of albumin $[15,16]$. In addition, for individuals with diabetes only, support comes from studies using strain-gauge plethysmography following forearm ischemia [17], and

Journal of Hypertension 2018, 36:1178-1187

aDivision of Nephrology, Department of Internal Medicine, Maastricht University Medical Center+, ${ }^{b}$ NUTRIM School of Nutrition and Translational Research in Metabolism, Maastricht University, 'Department of Internal Medicine, Maastricht University Medical Center+, ${ }^{\mathrm{d} C A R I M}$ School for Cardiovascular Diseases, Maastricht University, ${ }^{e}$ Department of Ophthalmology, Maastricht University Medical Center+, ${ }^{f}$ CAPHRI School for Public Health and Primary Care, ${ }^{9}$ Department of Epidemiology, Maastricht University and ${ }^{\mathrm{h}}$ Heart and Vascular Centre, Maastricht University Medical Center+, Maastricht, the Netherlands

Correspondence to Professor $\mathrm{Dr}$ Coen D.A. Stehouwer, Department of Internal Medicine, Maastricht University Medical Center+, P. Debyelaan 25, PO Box 5800, 6202AZ, Maastricht, the Netherlands. Tel: +31 433877006; fax: +31 433875006; e-mail: cda.stehouwer@mumc.nl

Received 11 September 2017 Revised 13 December 2017 Accepted 18 December 2017

J Hypertens 36:1178-1187 Copyright (C) 2018 Wolters Kluwer Health, Inc. All rights reserved.

DOI:10.1097/HJH.0000000000001674 
laser Doppler flowmetry following either iontophoresis of acetylcholine and sodium nitroprusside [18], or arterial occlusion [19].

Importantly, experimental data suggest that the association of microvascular endothelial dysfunction with albuminuria may be stronger in individuals with diabetes than in nondiabetic individuals [20,21].

In view of the above, we examined, in a populationbased cohort study of individuals without and with type 2 diabetes, whether direct measures of microvascular endothelial dysfunction are associated with albuminuria. For this purpose, we assessed flicker light-induced retinal arteriolar dilation and heat-induced skin hyperemia. These responses are thought to involve the endothelial release of NO as well as other vasodilators [22,23], and can be assessed noninvasively $[22,23]$ and reproducibly $[24,25]$ with the Dynamic Vessel Analyzer (DVA) [22] and laser Doppler flowmetry [23], respectively. Notwithstanding these similarities, both methods provide information on different vessel types and allow testing of vessel-reactivity to different stimuli and of different vessel responses. In addition, we examined whether any association of microvascular endothelial dysfunction with albuminuria is stronger in individuals with type 2 diabetes than in those without.

\section{RESEARGH DESIGN AND METHODS}

\section{The Maastricht Study population and design}

We used data from the Maastricht Study, an observational prospective population-based cohort study. The rationale and methodology have been described previously [26]. In brief, the study focuses on the etiology, pathophysiology, complications and comorbidities of type 2 diabetes, and is characterized by an extensive phenotyping approach. Eligible for participation were all individuals aged between 40 and 75 years and living in the southern part of the Netherlands. Participants were recruited through mass media campaigns and from the municipal registries and the regional Diabetes Patient Registry via mailings. Recruitment was stratified according to known type 2 diabetes status, with an oversampling of individuals with type 2 diabetes, for reasons of efficiency. The present report includes cross-sectional data from the first 3451 participants, who completed the baseline survey between November 2010 and September 2013. The examinations of each participant were performed within a time window of 3 months. The study has been approved by the institutional medical ethical committee (NL31329.068.10) and the Minister of Health, Welfare and Sports of the Netherlands (Permit 131088-105234-PG), and was conducted in accordance with the Declaration of Helsinki. All participants gave written informed consent.

\section{Assessment of microvascular endothelial function}

All participants were requested to refrain from smoking and drinking caffeine-containing beverages $3 \mathrm{~h}$ before the measurements. A light meal (breakfast and/or lunch) low in fat content was allowed at least $90 \mathrm{~min}$ before the start of the measurements [27].

\section{Flicker light-induced retinal arteriolar dilation}

The retinal arteriolar response to flicker light was assessed with the DVA (Imedos, Jena, Germany) as described previously [27]. Briefly, pupils were dilated with $0.5 \%$ tropicamide and $2.5 \%$ phenylephrine at least $15 \mathrm{~min}$ prior to the start of the examination. For safety reasons, participants with an intraocular pressure more than $30 \mathrm{mmHg}$ were excluded from the measurements. Per participant, either the left or right eye was selected depending on the time of day the measurement was performed and without reference to participant characteristics. A straight arteriolar segment of approximately $1.5 \mathrm{~mm}$ in length located 0.5-2 disc diameter from the margin of the optic disc in the temporal section was examined. Vessel diameter was automatically and continuously measured for $150 \mathrm{~s}$. A baseline recording of $50 \mathrm{~s}$ was followed by a 40-s flicker light exposure period (flicker frequency $12.5 \mathrm{~Hz}$, bright-todark contrast ratio 25:1), followed by a 60-s recovery period. Baseline retinal arteriolar diameter and flicker light-induced retinal arteriolar dilation were automatically calculated with the integrated DVA software (version 4.51; Imedos, Jena, Germany). Baseline retinal arteriolar diameter was calculated as the average diameter of the 20-50 s recording and was expressed in measurement units, where one measurement unit is equal to $1 \mu \mathrm{m}$ of the Gullstrand eye. Flicker light-induced retinal arteriolar dilation was expressed as the percentage retinal arteriolar dilation over baseline and based on the average dilation achieved at time-points 10 and $40 \mathrm{~s}$ during the flicker stimulation period.

\section{Heat-induced skin hyperemia}

Heat-induced skin hyperemia was assessed with a laser Doppler system (Periflux 5000; Perimed, Stockholm, Sweden), equipped with a thermostatic laser Doppler probe (PF457; Perimed) at the dorsal side of the wrist of the left hand, as described previously [27]. Briefly, skin blood flow measurements were performed in a climatecontrolled room at $24^{\circ} \mathrm{C}$. The laser Doppler output was recorded for 25 min with a sample rate of $32 \mathrm{~Hz}$, which gives semi-quantitative assessment of skin blood flow expressed in arbitrary perfusion units. Skin blood flow was first recorded unheated for 2 min to serve as a baseline. Thereafter, the temperature of the probe was rapidly and locally increased to $44^{\circ} \mathrm{C}$, and then kept constant until the end of the registration. In 596 individuals, heatinduced skin hyperemia measurements were recorded between 20 and $25 \mathrm{~min}$. These data were extrapolated to $25 \mathrm{~min}$ by adding the product of the average arbitrary perfusion units during the last completely recorded 1 -min interval and a correction factor of 1.017 , to the average arbitrary perfusion units during the recorded time, and taking the recording time into account. The correction factor of 1.017 was the ratio of the average arbitrary perfusion units in the 20-25 min interval (based on all available data) relative to the $19-20 \mathrm{~min}$ interval. The heatinduced skin hyperemic response was expressed as the percentage increase in average arbitrary perfusion units during the 23 min heating phase over the average baseline arbitrary perfusion units. 


\section{Albuminuria}

To assess urinary albumin excretion (UAE), participants were requested to collect two 24-h urine collections (Supplemental Methods, http://links.lww.com/HJH/A898). UAE was expressed both as a continuous and as a categorical variable $(<15 \mathrm{mg} / 24 \mathrm{~h}, 15$ to $<30 \mathrm{mg} / 24 \mathrm{~h}$, and $\geq 30 \mathrm{mg} / 24 \mathrm{~h}$ ), in agreement with the fact that an association with (cardiovascular) disease already exists below the clinical cut-off value of $30 \mathrm{mg} / 24 \mathrm{~h}$ [1]. These definitions were preferably based on the average of two (available in 91.5\% of the participants) 24-h urine collections.

\section{Potential confounders}

We assessed fasting glucose, hemoglobin A1c (HbA1c), glucose metabolism status, total cholesterol, high-density lipoprotein (HDL) cholesterol, low-density lipoprotein (LDL) cholesterol, triglycerides, BMI, waist circumference, office blood pressure, 24-h average ambulatory blood pressure, medication use, smoking behavior, alcohol consumption, educational level, prevalent CVD, and selfreported physical activity, as described previously [26]. Glomerular filtration rate (GFR) was estimated with the Chronic Kidney Disease Epidemiology Collaboration (CKD-EPI) equation based on both serum creatinine and serum cystatin C $\left(\mathrm{eGFR}_{\text {crcys }}\right)$ [28]. Further details on the definitions of the above potential confounders are provided in the Supplemental Methods, http://links.lww.com/HJH/ A898. In addition, we assessed the retinopathy status as described in the Supplemental Methods, http://links.lww. com/HJH/A898.

\section{Statistical analyses}

All analyses were performed with IBM SPSS Statistics Version 22.0 (IBM Corp, Armonk, New York, USA) in the population with complete data on all variables used in the fully adjusted model. Characteristics of the entire study population and according to albuminuria categories were summarized as means with standard deviations (SD), medians with interquartile ranges, and numbers with percentages, as appropriate.

The correlation between flicker light-induced retinal arteriolar dilation and heat-induced skin hyperemia was assessed with a Pearson correlation coefficient.

Baseline retinal arteriolar diameter and flicker lightinduced retinal arteriolar dilation were categorized into quartiles as the associations of flicker light-induced retinal arteriolar dilation with albuminuria seemed to be nonlinear. The quartile with the largest baseline retinal arteriolar diameter and the quartile with the greatest flicker lightinduced retinal arteriolar dilation served as reference categories. Baseline skin perfusion and heat-induced skin hyperemia were analyzed as continuous variables and expressed per one arbitrary perfusion unit lower baseline skin perfusion and per 100 percentage points lower heatinduced skin hyperemia, respectively.

Associations of baseline retinal arteriolar diameter, flicker light-induced retinal arteriolar dilation, baseline skin perfusion, and heat-induced skin hyperemia with UAE, expressed as a continuous variable, were examined with multivariable linear regression analyses. UAE was highly positively skewed and had to be transformed by taking the inverse square root to fulfill the normality assumption of linear regression. However, as the results obtained with natural logarithm transformed UAE were qualitatively similar and are more easily interpretable, these results are presented. The regression coefficients were exponentiated to obtain the ratio of UAE per one unit increase in the independent variable. In addition, we performed multinomial logistic regression analyses to examine the associations with UAE expressed as a categorical dependent variable ( $<15 \mathrm{mg} / 24 \mathrm{~h}$ served as the reference category). All analyses were adjusted for potential confounders as follows: model 1, unadjusted model; model 2, age, sex, glucose metabolism status; model $3 \mathrm{a}$, model 2 combined with waist circumference, total-to-HDL cholesterol ratio, triglycerides, use of lipid-modifying medication, office SBP, use of antihypertensive medication (including renin-angiotensin

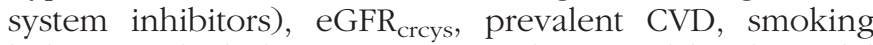
behavior, alcohol consumption, educational level; model $3 \mathrm{~b}$, as model $3 \mathrm{a}$ but adjusted for 24-h average ambulatory SBP instead of office SBP.

In addition, we tested for statistical interaction with type 2 diabetes $\left(P_{\text {interaction }}<0.10\right.$ was considered statistically significant). In these analyses, participants with normal glucose metabolism and prediabetes (Supplemental Methods, http://links.lww.com/HJH/A898) were combined into one category, because of the small number of participants with prediabetes and the even smaller number of participants in the respective albuminuria categories in this group.

We performed several additional analyses, each starting from model $3 a$. First, we tested for statistical interaction with HbA1c and eGFR to explore whether hyperglycemia and glomerular hyperfiltration [29,30], respectively, modified our results. Second, we performed several analyses to assess the robustness of our results. These are described in the Supplemental Methods, http://links.lww.com/HJH/ A898.

\section{RESULTS}

\section{Characteristics of the study population}

Supplemental Figure 1, http://links.lww.com/HJH/A898 is a flow diagram delineating the derivation of the final study populations. In total, 2095 participants were included in the analyses on flicker light-induced retinal arteriolar dilation (retina study population) and 1508 in the analyses on heat-induced skin hyperemia (skin study population). None of the participants was on dialysis. The characteristics shown in Table 1 of included versus excluded participants because of missing data were largely comparable (data not shown).

Table 1 and Supplemental Table 1, http://links.lww. $\mathrm{com} / \mathrm{HJH} / \mathrm{A} 898$ present the characteristics of the retina and skin study populations, respectively, according to albuminuria categories. The characteristics of both study populations were similar. In general, participants with higher UAE were older and more often men; more often had type 2 diabetes, hypertension and retinopathy; had a lower eGFR based on both serum creatinine and serum cystatin $\mathrm{C}\left(\mathrm{eGFR}_{\text {crcys }}\right)$ and a worse CVD risk profile; and 
TABLE 1. Characteristics of the retina study population stratified according to albuminuria categories

\begin{tabular}{|c|c|c|c|c|}
\hline Characteristic & $\begin{array}{l}\text { Entire population } \\
\qquad(n=2095)\end{array}$ & $\begin{array}{l}\text { Less than } 5 \mathrm{mg} / 24 \mathrm{~h} \\
\qquad(n=1707)\end{array}$ & $\begin{array}{l}15 \text { to less than } 30 \mathrm{mg} / 24 \mathrm{~h} \\
(n=216)\end{array}$ & $\begin{array}{l}\text { At least } 30 \mathrm{mg} / 24 \mathrm{~h} \\
\qquad(n=172)\end{array}$ \\
\hline \multicolumn{5}{|l|}{ Demographics } \\
\hline Age (years) & $59.7 \pm 8.3$ & $59.1 \pm 8.2$ & $61.7 \pm 8.4$ & $63.3 \pm 7.6$ \\
\hline Men & $1075(51.3 \%)$ & $824(48.3 \%)$ & $129(59.7 \%)$ & $122(70.9 \%)$ \\
\hline \multicolumn{5}{|l|}{ Educational level } \\
\hline Low & $332(15.8 \%)$ & $247(14.5 \%)$ & $36(16.7 \%)$ & $49(28.5 \%)$ \\
\hline Middle & $898(42.9 \%)$ & $745(43.6 \%)$ & $93(43.1 \%)$ & $60(34.9 \%)$ \\
\hline High & $865(41.3 \%)$ & $715(41.9 \%)$ & $87(40.3 \%)$ & $63(36.6 \%)$ \\
\hline Prior cardiovascular disease & $328(15.7 \%)$ & $239(14.0 \%)$ & $39(18.1 \%)$ & $50(29.1 \%)$ \\
\hline \multicolumn{5}{|l|}{ Lifestyle variables } \\
\hline \multicolumn{5}{|l|}{ Smoking behaviour } \\
\hline Never smoker & $737(35.2 \%)$ & $630(36.9 \%)$ & $64(29.6 \%)$ & $43(25.0 \%)$ \\
\hline Former smoker & $1104(52.7 \%)$ & $881(51.6 \%)$ & $126(58.3 \%)$ & $97(56.4 \%)$ \\
\hline Current smoker & $254(12.1 \%)$ & $196(11.5 \%)$ & $26(12.0 \%)$ & $32(18.6 \%)$ \\
\hline \multicolumn{5}{|l|}{ Alcohol consumption } \\
\hline None & $377(18.0 \%)$ & $294(17.2 \%)$ & $36(16.7 \%)$ & $47(27.3 \%)$ \\
\hline $\begin{array}{l}\text { Low (women, } \leq 7 \text { glasses/week; } \\
\text { men, } \leq 14 \text { glasses/week) }\end{array}$ & $1188(56.7 \%)$ & $979(57.4 \%)$ & $124(57.4 \%)$ & $85(49.4 \%)$ \\
\hline $\begin{array}{l}\text { High (women }>7 \text { glasses/week; } \\
\text { men }>14 \text { glasses/week) }\end{array}$ & $530(25.3 \%)$ & $434(25.4 \%)$ & $56(25.9 \%)$ & $40(23.3 \%)$ \\
\hline \multicolumn{5}{|l|}{ Metabolic variables } \\
\hline \multicolumn{5}{|l|}{ BMI categories ${ }^{a}$} \\
\hline Normal weight $\left(<25 \mathrm{~kg} / \mathrm{m}^{2}\right)$ & $759(36.2 \%)$ & $674(39.5 \%)$ & $52(24.1 \%)$ & $33(19.2 \%)$ \\
\hline Overweight $\left(25\right.$ to $<30 \mathrm{~kg} / \mathrm{m}^{2}$ ) & $890(42.5 \%)$ & $714(41.9 \%)$ & $104(48.1 \%)$ & $72(41.9 \%)$ \\
\hline Obesity $\left(\geq 30 \mathrm{~kg} / \mathrm{m}^{2}\right)$ & $445(21.3 \%)$ & $318(18.6 \%)$ & $60(27.8 \%)$ & $67(39.0 \%)$ \\
\hline \multicolumn{5}{|l|}{ Waist circumference $(\mathrm{cm})$} \\
\hline Men & $101.1 \pm 11.8$ & $99.7 \pm 11.3$ & $104.4 \pm 10.7$ & $106.8 \pm 13.8$ \\
\hline Women & $89.3 \pm 12.6$ & $88.5 \pm 12.0$ & $91.8 \pm 14.6$ & $97.7 \pm 15.2$ \\
\hline Office SBP (mmHg) & $135.0 \pm 18.0$ & $133.3 \pm 17.4$ & $140.4 \pm 17.7$ & $144.6 \pm 19.7$ \\
\hline Office DBP (mmHg) & $76.4 \pm 9.9$ & $76.0 \pm 9.8$ & $77.8 \pm 10.6$ & $78.0 \pm 9.6$ \\
\hline 24-h average ambulatory SBP $(\mathrm{mmHg})^{\mathrm{a}}$ & $119.1 \pm 11.6$ & $117.8 \pm 10.9$ & $123.5 \pm 11.9$ & $126.7 \pm 13.2$ \\
\hline 24-h average ambulatory DBP $(\mathrm{mmHg})^{\mathrm{a}}$ & $73.5 \pm 7.2$ & $73.2 \pm 7.0$ & $75.1 \pm 8.4$ & $74.8 \pm 7.4$ \\
\hline Hypertension & $1166(55.7 \%)$ & $871(51.0 \%)$ & $151(69.9 \%)$ & $144(83.7 \%)$ \\
\hline \multicolumn{5}{|l|}{ Glucose metabolism status } \\
\hline Normal glucose metabolism & $1197(57.1 \%)$ & $1053(61.7 \%)$ & $91(42.1 \%)$ & $53(30.8 \%)$ \\
\hline Impaired fasting glucose & $85(4.1 \%)$ & $70(4.1 \%)$ & $10(4.6 \%)$ & $5(2.9 \%)$ \\
\hline Impaired glucose tolerance & $239(11.4 \%)$ & $206(12.1 \%)$ & $20(9.3 \%)$ & $13(7.6 \%)$ \\
\hline Type 2 diabetes & $574(27.4 \%)$ & $378(22.1 \%)$ & $95(44.0 \%)$ & $101(58.7 \%)$ \\
\hline \multicolumn{5}{|l|}{ Fasting glucose $(\mathrm{mmol} / \mathrm{l})$} \\
\hline Without type 2 diabetes & $5.3 \pm 0.5$ & $5.3 \pm 0.5$ & $5.4 \pm 0.6$ & $5.5 \pm 0.6$ \\
\hline With type 2 diabetes & $8.0 \pm 2.1$ & $7.8 \pm 1.9$ & $7.9 \pm 2.2$ & $8.6 \pm 2.6$ \\
\hline \multicolumn{5}{|l|}{$\operatorname{HbA} 1 \mathrm{C}(\%)^{a}$} \\
\hline Without type 2 diabetes & $5.5 \pm 0.4$ & $5.5 \pm 0.4$ & $5.5 \pm 0.4$ & $5.5 \pm 0.3$ \\
\hline With type 2 diabetes & $6.9 \pm 1.1$ & $6.8 \pm 0.9$ & $6.9 \pm 1.1$ & $7.3 \pm 1.4$ \\
\hline Total cholesterol (mmol/l) & $5.2 \pm 1.2$ & $5.3 \pm 1.2$ & $5.0 \pm 1.2$ & $4.8 \pm 1.2$ \\
\hline \multicolumn{5}{|l|}{ HDL cholesterol (mmol/l) } \\
\hline Men & $1.3 \pm 0.4$ & $1.4 \pm 0.4$ & $1.3 \pm 0.3$ & $1.3 \pm 0.4$ \\
\hline Women & $1.8 \pm 0.5$ & $1.8 \pm 0.5$ & $1.7 \pm 0.5$ & $1.6 \pm 0.5$ \\
\hline LDL cholesterol (mmol/l) & $3.1 \pm 1.0$ & $3.1 \pm 1.0$ & $2.9 \pm 1.0$ & $2.6 \pm 1.1$ \\
\hline Triglycerides $(\mathrm{mmol} / \mathrm{l})$ & $1.22[0.88-1.72]$ & $1.18[0.86-1.66]$ & $1.31[0.97-1.90]$ & $1.49[1.10-2.23]$ \\
\hline Total-to-HDL cholesterol ratio & $3.6 \pm 1.2$ & $3.6 \pm 1.2$ & $3.7 \pm 1.2$ & $3.7 \pm 1.1$ \\
\hline \multicolumn{5}{|l|}{ Kidney function } \\
\hline eGFR $\left(\mathrm{ml} / \mathrm{min}\right.$ per $\left.1.73 \mathrm{~m}^{2}\right)$ & $88.3 \pm 14.7$ & $89.1 \pm 13.8$ & $87.0 \pm 16.4$ & $81.8 \pm 18.1$ \\
\hline Urinary albumin excretion (mg/24h) & $6.5[3.9-11.6]$ & $5.4[3.6-8.1]$ & $19.3[16.4-23.4]$ & $62.3[40.5-109.6]$ \\
\hline \multicolumn{5}{|l|}{ Urinary albumin excretion categories } \\
\hline$<15 \mathrm{mg} / 24 \mathrm{~h}$ & $1707(81.5 \%)$ & - & - & - \\
\hline 15 to $<30 \mathrm{mg} / 24 \mathrm{~h}$ & $216(10.3 \%)$ & - & - & - \\
\hline$\geq 30 \mathrm{mg} / 24 \mathrm{~h}$ & $172(8.2 \%)$ & - & - & - \\
\hline \multicolumn{5}{|l|}{ Medication } \\
\hline $\begin{array}{l}\text { Antihypertensive medication (including } \\
\text { renin-angiotensin system inhibitors) }\end{array}$ & $806(38.5 \%)$ & $587(34.4 \%)$ & $101(46.8 \%)$ & $118(68.6 \%)$ \\
\hline Renin-angiotensin system inhibitor & $604(28.8 \%)$ & $424(24.8 \%)$ & $80(37.0 \%)$ & $100(58.1 \%)$ \\
\hline Lipid-modifying medication & $737(35.2 \%)$ & $541(31.7 \%)$ & $94(43.5 \%)$ & $102(59.3 \%)$ \\
\hline Microvascular measurements & & & & \\
\hline Retinopathy ${ }^{a}$ & $27(1.3 \%)$ & $16(1.0 \%)$ & $6(3.0 \%)$ & $5(3.0 \%)$ \\
\hline $\begin{array}{l}\text { Baseline retinal arteriolar diameter } \\
\text { (measurement units) }\end{array}$ & $115.5 \pm 15.6$ & $115.6 \pm 15.7$ & $115.5 \pm 16.0$ & $115.1 \pm 14.5$ \\
\hline Mean percentage arteriolar dilatation (\%) & $2.6[0.8-5.0]$ & $2.8[0.9-5.2]$ & $1.9[0.6-3.8]$ & $1.4[0.4-4.0]$ \\
\hline
\end{tabular}


TABLE 1 (Continued)

\begin{tabular}{|c|c|c|c|c|}
\hline Characteristic & $\begin{array}{l}\text { Entire population } \\
\qquad(n=2095)\end{array}$ & $\begin{array}{l}\text { Less than } 5 \mathrm{mg} / 24 \mathrm{~h} \\
\qquad(n=1707)\end{array}$ & $\begin{array}{l}15 \text { to less than } 30 \mathrm{mg} / 24 \mathrm{~h} \\
\qquad(n=216)\end{array}$ & $\begin{array}{l}\text { At least } 30 \mathrm{mg} / 24 \mathrm{~h} \\
(n=172)\end{array}$ \\
\hline $\begin{array}{l}\text { Baseline skin perfusion (perfusion units) })^{a} \\
\text { Heat-induced skin hyperemia (\%) }\end{array}$ & $\begin{array}{c}9.3[7.1-13.1] \\
998.5[588.5-1507.2]\end{array}$ & $\begin{array}{c}9.2[7.0-12.8] \\
1028.3[613.5-1515.4]\end{array}$ & $\begin{array}{c}9.5[7.4-14.9] \\
878.4[457.4-1502.5]\end{array}$ & $\begin{array}{c}9.2[7.4-13.4] \\
785.3[468.4-1137.3]\end{array}$ \\
\hline
\end{tabular}

Data are presented as $n(\%)$, mean $\pm \mathrm{SD}$, or median [interquartile range]

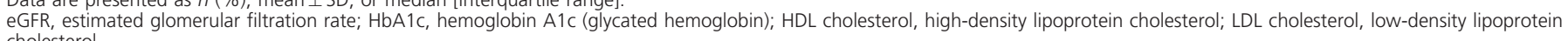
cholesterol

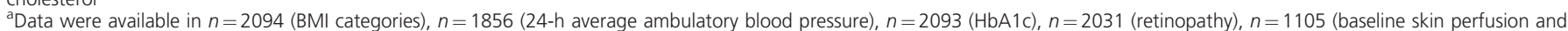
heat-induced skin hyperemia).

more often used antihypertensive and lipid-modifying medication.

\section{Flicker light-induced retinal arteriolar dilation and heat-induced skin hyperemia}

The Pearson correlation coefficient between retinal arteriolar dilation and heat-induced skin hyperemia was 0.05 $(P=0.121)$.

\section{Flicker light-induced retinal arteriolar dilation and albuminuria}

Flicker light-induced retinal arteriolar dilation was categorized into quartiles as its association with albuminuria seemed to be nonlinear. Quartile (Q) 1 consisted of the participants with the greatest flicker light-induced retinal arteriolar dilation and served as the reference category. In the entire retina study population and with UAE expressed as a continuous variable, UAE was higher in Q2 (albeit not statistically significantly), was statistically significantly higher in Q3 and then showed no further increase in Q4, after adjustment for potential confounders (Table 2, model 3a). For example, UAE was [ratio (95\% confidence interval: 95\% CI)] $1.099(0.987-1.224)$ times higher in Q2, 1.189 (1.067-1.326) times higher in Q3, and 1.168
(1.046-1.303) times higher in Q4. Results were similar whenever we replaced office SBP with 24-h average ambulatory SBP ( $n=1856$; Table 2, model $3 \mathrm{~b}$ ) and whenever UAE was expressed as a categorical variable (Supplemental Table 2, http://links.lww.com/HJH/A898).

Analyses with interaction terms suggested that the association of flicker light-induced retinal arteriolar dilation with albuminuria was stronger in participants with type 2 diabetes than without type 2 diabetes ( $P$ value of the interaction term $\left(P_{\text {interaction }}\right)<0.10$ for the associations of Q3 and Q4 with UAE expressed as a continuous variable, and for the association of Q3 with UAE $\geq 30 \mathrm{mg} / 24 \mathrm{~h}$; Table 2 and Supplemental Table 2, http://links.lww.com/ HJH/A898, model 3a; also illustrated in Fig. 1a). However, the stratified analyses were hampered by a loss of statistical power.

Baseline retinal arteriolar diameter was not associated with continuous or categorical UAE (Supplemental Tables 3 and 4, http://links.lww.com/HJH/A898, models 1-3), regardless of type 2 diabetes $\left(P_{\text {interaction }}>0.10\right)$.

\section{Heat-induced skin hyperemia and albuminuria}

In the entire skin study population, heat-induced skin hyperemia was not associated with UAE, expressed as

TABLE 2. Associations of flicker light-induced retinal arteriolar dilation with urinary albumin excretion expressed as a continuous variable

\begin{tabular}{|c|c|c|c|c|c|c|c|c|}
\hline \multirow[b]{2}{*}{ Model } & & \multicolumn{2}{|c|}{$\begin{array}{c}\text { Entire retina study } \\
\text { population }\end{array}$} & \multicolumn{2}{|c|}{$\begin{array}{l}\text { Without type } \\
2 \text { diabetes }\end{array}$} & \multicolumn{2}{|c|}{$\begin{array}{l}\text { With type } \\
2 \text { diabetes }\end{array}$} & \multirow[b]{2}{*}{$\boldsymbol{P}_{\text {interaction }}$} \\
\hline & & Ratio $(95 \% \mathrm{Cl})$ & $P$ value & Ratio $(95 \% \mathrm{Cl})$ & $P$ value & Ratio $(95 \% \mathrm{Cl})$ & $P$ value & \\
\hline \multirow[t]{4}{*}{1} & Q1 & Reference & & Reference & & Reference & & \\
\hline & Q2 & $1.110(0.989 ; 1.245)$ & 0.075 & $1.037(0.915 ; 1.174)$ & 0.575 & $1.302(1.020 ; 1.664)$ & 0.034 & 0.102 \\
\hline & Q3 & $1.306(1.164 ; 1.465)$ & $<0.001$ & $1.120(0.985 ; 1.271)$ & 0.084 & $1.649(1.307 ; 2.079)$ & $<0.001$ & 0.004 \\
\hline & Q4 & $1.370(1.221 ; 1.537)$ & $<0.001$ & $1.154(1.011 ; 1.317)$ & 0.033 & $1.536(1.229 ; 1.919)$ & $<0.001$ & 0.031 \\
\hline \multirow[t]{4}{*}{2} & Q1 & Reference & & Reference & & Reference & & \\
\hline & Q2 & $1.091(0.977 ; 1.217)$ & 0.122 & $1.052(0.931 ; 1.190)$ & 0.415 & $1.292(1.014 ; 1.644)$ & 0.038 & 0.140 \\
\hline & Q3 & $1.203(1.077 ; 1.343)$ & 0.001 & $1.111(0.978 ; 1.260)$ & 0.105 & $1.584(1.259 ; 1.994)$ & $<0.001$ & 0.008 \\
\hline & Q4 & $1.179(1.054 ; 1.318)$ & 0.004 & $1.115(0.978 ; 1.271)$ & 0.103 & $1.458(1.170 ; 1.818)$ & 0.001 & 0.040 \\
\hline \multirow[t]{4}{*}{ 3а } & Q1 & Reference & & Reference & & Reference & & \\
\hline & Q2 & $1.099(0.987 ; 1.224)$ & 0.086 & $1.062(0.941 ; 1.198)$ & 0.331 & $1.279(1.008 ; 1.623)$ & 0.043 & 0.170 \\
\hline & Q3 & $1.189(1.067 ; 1.326)$ & 0.002 & $1.106(0.976 ; 1.252)$ & 0.112 & $1.520(1.214 ; 1.906)$ & $<0.001$ & 0.015 \\
\hline & Q4 & $1.168(1.046 ; 1.303)$ & 0.006 & $1.117(0.982 ; 1.271)$ & 0.092 & $1.387(1.116 ; 1.724)$ & 0.003 & 0.092 \\
\hline \multirow[t]{4}{*}{$3 b$} & Q1 & Reference & & Reference & & Reference & & \\
\hline & Q2 & $1.116(0.998 ; 1.247)$ & 0.054 & $1.066(0.941 ; 1.208)$ & 0.317 & $1.340(1.050 ; 1.713)$ & 0.019 & 0.102 \\
\hline & Q3 & $1.153(1.029 ; 1.289)$ & 0.013 & $1.084(0.953 ; 1.234)$ & 0.216 & $1.436(1.137 ; 1.813)$ & 0.002 & 0.039 \\
\hline & Q4 & $1.147(1.024 ; 1.285)$ & 0.018 & $1.100(0.963 ; 1.256)$ & 0.159 & $1.350(1.079 ; 1.690)$ & 0.009 & 0.123 \\
\hline
\end{tabular}

Ratios represent the ratio of urinary albumin excretion in the respective quartile of flicker light-induced retinal arteriolar dilation compared with the reference quartile. Model 1 :

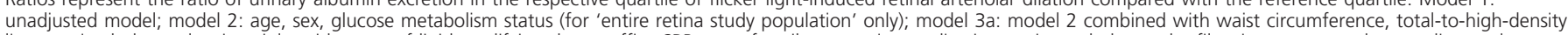
lipoprotein cholesterol ratio, triglycerides, use of lipid-modifying drugs, office SBP, use of antihypertensive medication, estimated glomerular filtration rate, prevalent cardiovascular

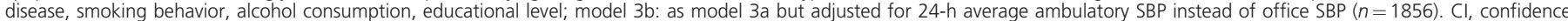
interval; Q, quartile. 
(a)

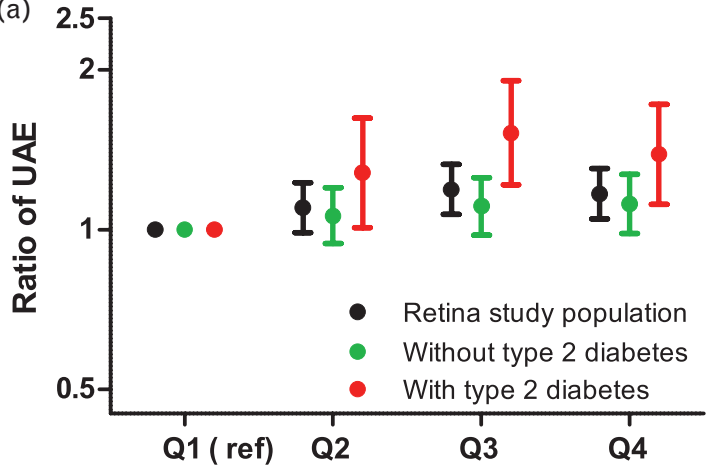

Flicker light-induced retinal arteriolar dilation (\%) (b)

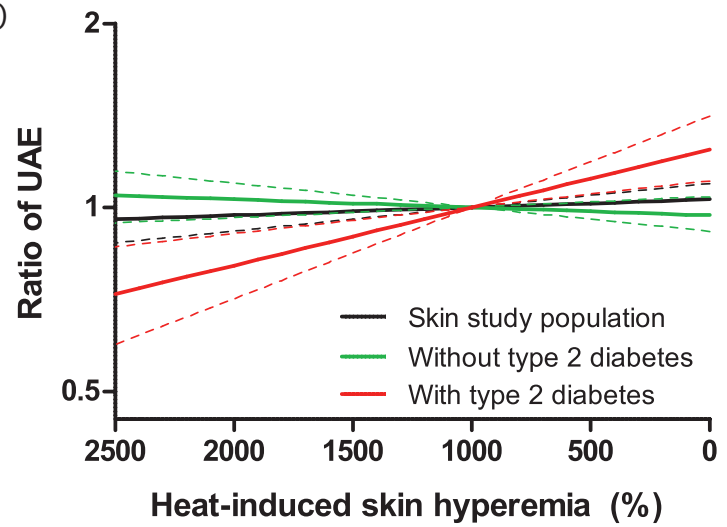

(c)

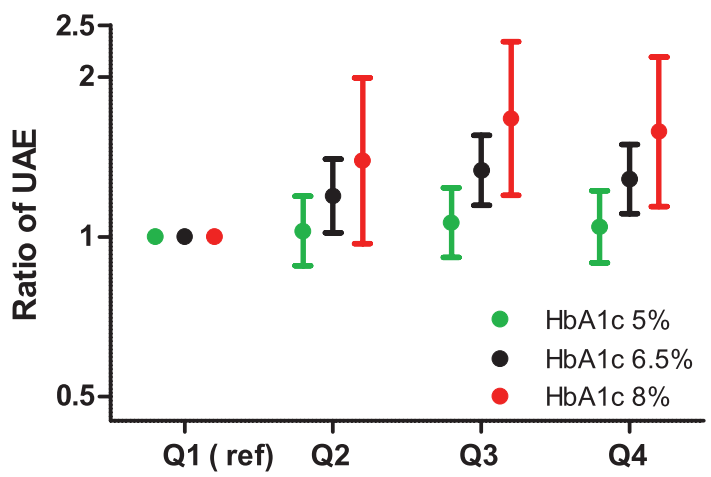

Flicker light-induced retinal arteriolar dilation (\%) (d)

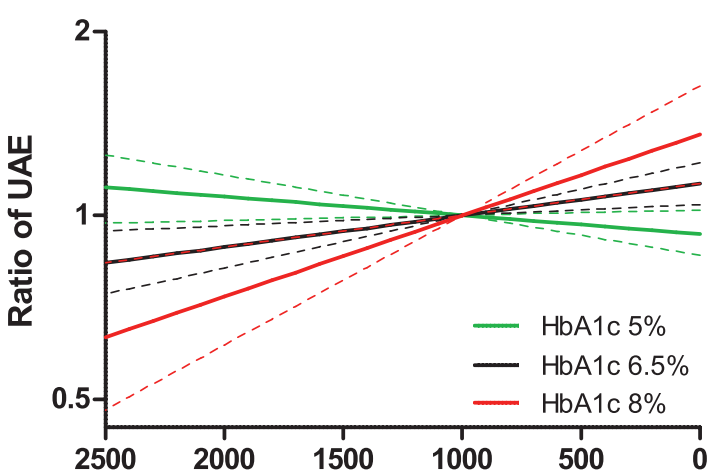

Heat-induced skin hyperemia (\%)

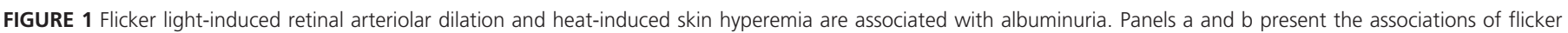
light-induced retinal arteriolar dilation and heat-induced skin hyperemia, respectively, with albuminuria, and the interaction with type 2 diabetes. Panels $c$ and $d$ present the interaction of flicker light-induced retinal arteriolar dilation and heat-induced skin hyperemia, respectively, with HbA1c. Ratios indicate the ratio of geometric mean

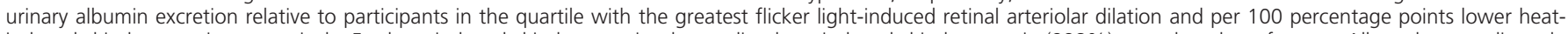
induced skin hyperemia, respectively. For heat-induced skin hyperemia, the median heat-induced skin hyperemia (998\%) served as the reference. All results are adjusted for the variables of model 3a (see text). Bars and dashed lines indicate 95\% confidence intervals. ref, reference; HbA1C, hemoglobin A1c (glycated hemoglobin); UAE, urinary albumin excretion.

either a continuous or a categorical variable, after adjustment for potential confounders (Table 3 and Supplemental Table 5, http://links.lww.com/HJH/A898, model 3a). However, analyses with interaction terms suggested statistical interaction with type 2 diabetes $\left(P_{\text {interaction }}<0.10\right)$, to such an extent that lower heat-induced skin hyperemia was associated with higher UAE, expressed as a continuous variable, in participants with type 2 diabetes but not in those without type 2 diabetes (Table 3, models 1-3; also illustrated in Fig. 1b). For example, after adjustment for potential confounders and in participants with type 2 diabetes, each 100 percentage points lower heat-induced skin hyperemia was associated with a [Ratio (95\% CI)] 1.022 (1.010-1.035) times higher UAE (Table 3, model 3a). Results were similar whenever we replaced office SBP with 24-h average ambulatory SBP ( $n=1324$; Table 3 , model 3b) and whenever UAE was expressed as a categorical variable (Supplemental Table 5, http://links.lww.com/HJH/A898).

In the entire skin study population, baseline skin perfusion was associated with UAE expressed as a categorical variable, but not with UAE expressed as a continuous variable (Supplemental Tables 6 and 7, http://links.lww.com/

TABLE 3. Associations of heat-induced skin hyperemia with urinary albumin excretion expressed as a continuous variable

\begin{tabular}{|c|c|c|c|c|c|c|c|}
\hline \multirow[b]{2}{*}{ Model } & \multicolumn{2}{|c|}{ Entire skin study population } & \multicolumn{2}{|c|}{ Without type 2 diabetes } & \multicolumn{2}{|c|}{ With type 2 diabetes } & \multirow[b]{2}{*}{$P_{\text {interaction }}$} \\
\hline & Ratio $(95 \% \mathrm{Cl})$ & $P$ value & Ratio $(95 \% \mathrm{Cl})$ & $P$ value & Ratio $(95 \% \mathrm{Cl})$ & $P$ value & \\
\hline 1 & $1.013(1.007 ; 1.019)$ & $<0.001$ & $1.001(0.994 ; 1.008)$ & 0.839 & $1.027(1.015 ; 1.040)$ & $<0.001$ & $<0.001$ \\
\hline 2 & $1.004(0.998 ; 1.010)$ & 0.217 & $0.997(0.990 ; 1.004)$ & 0.447 & $1.024(1.012 ; 1.037)$ & $<0.001$ & $<0.001$ \\
\hline $3 a$ & $1.003(0.997 ; 1.009)$ & 0.264 & $0.997(0.991 ; 1.004)$ & 0.467 & $1.022(1.010 ; 1.035)$ & $<0.001$ & $<0.001$ \\
\hline $3 b$ & $1.002(0.996 ; 1.008)$ & 0.590 & $0.996(0.989 ; 1.003)$ & 0.287 & $1.019(1.007 ; 1.031)$ & 0.002 & 0.001 \\
\hline
\end{tabular}

Ratios represent the ratio of urinary albumin excretion per 100 percentage points lower heat-induced skin hyperemia. Model 1: unadjusted model; model 2: age, sex, glucose

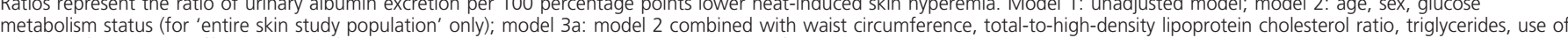
lipid-modifying drugs, office SBP, use of antihypertensive medication, estimated glomerular filtration rate, prevalent cardiovascular disease, smoking behavior, alcohol consumption, educational level; model 3b: as model 3a but adjusted for 24-h average ambulatory SBP instead of office SBP $(n=1324)$. Cl, confidence interval. 
HJH/A898, models 1-3). Analyses with interaction terms suggested that the association with UAE expressed as a continuous variable was stronger in participants with type 2 diabetes $\left(P_{\text {interaction }}=0.10\right)$, although this was not clear for UAE expressed as a categorical variable (Supplemental Tables 6 and 7, http://links.lww.com/HJH/A898).

\section{Additional analyses}

First, we explored whether hyperglycemia and glomerular hyperfiltration $[29,30]$ could explain why the associations of flicker light-induced retinal arteriolar dilation and heatinduced skin hyperemia with albuminuria were more evident in participants with type 2 diabetes. Therefore, we additionally tested for statistical interaction between both measures of microvascular endothelial dysfunction and hemoglobin A1c (HbA1c; glycated hemoglobin) as well as eGFR in model $3 \mathrm{a}$. These analyses indicated that flicker light-induced retinal arteriolar dilation and heat-induced skin hyperemia were more strongly associated with albuminuria at higher HbA1c levels $\left(P_{\text {interaction }}<0.10\right.$; illustrated in Fig. 1c and d), but not at higher eGFR (data not shown). Thus, for Q2, Q3, and Q4 of flicker light-induced retinal arteriolar dilation, the ratio of UAE relative to Q1 was 1.106 $(P=0.186), 1.162(P=0.035)$, and $1.147(P=0.052)$ times higher, respectively, per one percentage point higher HbA1c. In addition, the ratio of UAE per 100 percentage points lower heat-induced skin hyperemia was 1.013 $(P=0.002)$ times higher per one percentage point higher HbA1c. Second, the results of additional analyses with alteration of the definitions of potential confounders, exploration of the presence of misclassification of albuminuria status, analyses with absolute versus percentage differences in retinal arteriolar diameter and skin perfusion, and analyses with quintiles and deciles of flicker light-induced retinal arteriolar dilation supported the robustness of our results (Supplemental Results, http://links.lww.com/HJH/ A898).

\section{DISGUSSION}

This population-based study, in individuals without and with type 2 diabetes, on the association of microvascular endothelial dysfunction with albuminuria showed that lower flicker light-induced retinal arteriolar dilation was associated with albuminuria and that this association was stronger in individuals with type 2 diabetes. In addition, lower heat-induced skin hyperemia was associated with albuminuria in participants with type 2 diabetes but not in nondiabetic participants. These associations were independent of CVD risk factors, including 24-h average ambulatory blood pressure. To our knowledge, this is the first population-based study that provides direct support for the concept that microvascular endothelial dysfunction related to albuminuria is extensive, if not generalized, and that this association is stronger in individuals with than in those without type 2 diabetes.

These results agree with those of studies in individuals with type 1 or type 2 diabetes, which have shown associations of direct measures of microvascular endothelial dysfunction in skin with albuminuria [17-19]. The present study expands this knowledge, as it is the first to examine microvascular endothelial dysfunction in two vascular beds in a large, well characterized population-based sample of individuals with and without type 2 diabetes, and with extensive adjustment for potential confounders.

The association of microvascular endothelial dysfunction with albuminuria fits the hypothesis that albuminuria is a biomarker of generalized endothelial dysfunction [6]. Endothelial dysfunction of the renal microcirculation may cause albuminuria by increasing glomerular capillary wall permeability and intraglomerular pressure [6]. The former may involve alterations in the barrier properties of the glomerular endothelium itself [6] and altered endothelial-podocyte cross-talk leading to podocyte injury [31]. However, not all studies observed an association between microvascular endothelial dysfunction and albuminuria. For example, in a study in individuals with hypertension, the endothelium-dependent vasodilatory response in the forearm bed was not associated with albuminuria [32]. This may be because of differences in participant selection (e.g. hypertension versus diabetes).

The molecular mechanisms involved in both flicker light-induced retinal arteriolar dilation and heat-induced skin hyperemia include the endothelial release of nitric oxide (NO) [22,23]. Reduced NO availability may reflect microvascular endothelial dysfunction in general, but may also play a direct role. Indeed, NO has been shown to preserve glomerular capillary wall permeability [33], and endothelial NO synthase inhibition induced podocyte injury and albuminuria in an animal model of diabetes [34]. In contrast, NO's role in the regulation of renal hemodynamics is unlikely to explain our results, because reduced $\mathrm{NO}$ availability is expected to reduce rather than increase intraglomerular pressure [35]. In addition to NO, other substances secreted by the endothelium may be involved in the association of microvascular endothelial dysfunction and albuminuria.

The correlation between flicker light-induced retinal arteriolar dilation and heat-induced skin hyperemia was weak and not statistically significant. This may have several explanations. First, both methods assess different vessel types (i.e. relatively large arterioles in the retina versus small arterioles, capillaries, and venules in skin) [36]. Second, both methods assess different outcomes (i.e. a direct stimulus-induced increase in vessel diameter in the retina versus an indirect estimate of vasodilation by measuring stimulusinduced increase in perfusion in skin) [22,23]. Third, both methods use different stimuli to elicit the responses (i.e. flicker light stimulation versus thermal stimulation) [22,23].

The results of this study suggest that hyperglycemia amplifies the association of microvascular endothelial dysfunction with albuminuria. This observation is in agreement with animal studies, which have shown higher UAE in NO synthase knockout models in the presence of diabetes $[20,21]$. In this regard, it has been suggested that hyperglycemia leads to dysregulation of the vascular endothelial growth factor (VEGF)-NO axis [37]. That is, high glucose levels may reduce NO availability and increase VEGF expression [37]. In the face of reduced NO availability, VEGF may exacerbate vascular injury through abnormal angiogenesis [37] and alteration of the endothelial glycocalyx [38]. Alternatively, (hyperglycemia-induced) 
glomerular hyperfiltration [29,30] may increase permeation of albumin through the injured glomerular capillary wall. Although this hypothesis was not supported by a positive interaction of microvascular endothelial dysfunction with eGFR, the low precision of the eGFR equations at higher GFR [28] may have masked this interaction.

Impaired neural activity is an additional mechanism that can reduce both flicker light-induced retinal arteriolar dilation and heat-induced skin hyperemia $[22,23]$ and may as well be involved in their association with albuminuria. For example, autonomic dysfunction may compromise autoregulation of intraglomerular pressure and has indeed been associated with albuminuria [39].

The association of flicker light-induced retinal arteriolar dilation with albuminuria leveled off at lower levels. This may be explained by selective nonparticipation of individuals with higher UAE and the lowest flicker light-induced retinal arteriolar dilation, who may suffer from the greatest burden of comorbid disease, including impaired cognitive performance and depression (i.e. incidence-prevalence bias). In addition, the 95\% CIs are too wide to exclude the possibility that the true association is linear.

Baseline retinal arteriolar diameter was not associated with albuminuria, whereas lower baseline skin perfusion was associated with lower albuminuria. However, the pattern of associations of baseline skin perfusion was not consistent and not biologically plausible. Therefore, we attribute these findings to the play of chance.

From a clinical perspective, microvascular endothelial dysfunction may explain, at least in part, associations of albuminuria with retinopathy, impaired cognitive performance, depression, and heart failure $[2,5,7,8]$. In this regard, the endothelial dysfunction observed in the retinal microcirculation of individuals with albuminuria may be particularly relevant to its associations with impaired cognitive performance and depression as well as retinopathy, as the retinal and cerebral microcirculation show embryological, anatomical, and physiological similarities [40]. In addition, the skin microcirculation has been suggested to mirror the systemic microcirculation $[41,42]$.

The results of this study also suggest that albuminuria may be a better biomarker of microvascular endothelial dysfunction in individuals with than in those without type 2 diabetes. Therefore, associations of albuminuria with microvascular disease may be stronger in type 2 diabetes. To the best of our knowledge, there are no published data on this issue.

An important strength of this study is the detailed characterization of its population, which allowed for adjustment for an extensive series of potential confounders. In particular, 24-h average ambulatory blood pressure may better capture the effects of blood pressure on albuminuria than office blood pressure, which reduces residual confounding. In addition, an extensive series of additional analyses verified the robustness of our results.

The present study also had some limitations. First, the cross-sectional design limited causal inferences. Second, despite adjustment for an extensive series of potential confounders, we cannot fully exclude residual confounding. Conversely, the inclusion of variables, which could also be intermediates in the association of microvascular endothelial dysfunction with albuminuria in our models may have introduced overadjustment bias [43]. For example, higher blood pressure may be both a cause and a consequence of endothelial dysfunction [44]. In addition, adjustment for CVD and retinopathy might be considered unnecessary as both may be manifestations of microvascular endothelial dysfunction [43]. Nevertheless, the regression coefficients were relatively robust to adjustment for the variables in model $3 \mathrm{a}$ and the variables in the additional analyses, which suggests that both residual confounding and overadjustment bias are limited. Third, data on flicker light-induced retinal arteriolar dilation and heat-induced skin hyperemia were not available in all participants (mainly because of logistic reasons [27]), and the retina and skin study populations did not entirely overlap. However, the characteristics of included participants and participants who were excluded because of missing data were largely comparable, as were the characteristics of the participants in the retina and skin study populations. Fourth, actual glycaemia levels were not assessed at the day of microvascular endothelial function assessment. Therefore, acute effects of hyperglycemia could not be taken into account. Fifth, the absolute number of participants with albuminuria 15 to less than $30 \mathrm{mg} / 24 \mathrm{~h}$ and at least $30 \mathrm{mg} / 24 \mathrm{~h}$ in this population-based study was relatively low. This may reduce statistical power, in particular for the stratified analyses and the supplemental analyses with albuminuria expressed as a categorical variable. Sixth, the study population primarily consisted of Caucasian individuals from European descent (98.9\%), which potentially limits its generalizability to other populations.

In conclusion, lower flicker light-induced retinal arteriolar dilation was independently associated with albuminuria and this association was stronger in individuals with type 2 diabetes. In addition, lower heat-induced skin hyperemia was independently associated with albuminuria in individuals with type 2 diabetes. Thus, this is the first population-based study that provides direct support for the concept that microvascular endothelial dysfunction related to albuminuria is extensive, if not generalized, and that this association is stronger in individuals with than in those without type 2 diabetes. This suggests that individuals with albuminuria may not only benefit from improving renal endothelial function and reducing albuminuria but also from improving systemic endothelial function as well.

\section{ACKNOWLEDGEMENTS}

The authors are grateful to the participants of The Maastricht Study and to all research assistants who were involved in the data acquisition. Perimed (Järfalla, Sweden) is gratefully acknowledged for their support.

The results of this study were presented at the Annual Meeting of the European Diabetic Nephropathy Study Group (Helsinki, 2017).

Author contributions: R.J.H.M. participated in data collection, analysed, and interpreted the data and wrote the manuscript. C.D.A.S. interpreted the data and contributed to writing the manuscript, and developed The Maastricht Study concept and protocol. A.J.H.M. contributed to data processing, interpreted the data and critically reviewed 
drafts of the manuscript. J.P.K. interpreted the data and critically reviewed drafts of the manuscript. R.M.A.H. interpreted the data and critically reviewed drafts of the manuscript, and developed The Maastricht Study concept and protocol. T.T.J.M.B., A.A.K., and J.S.A.G.S. contributed to data processing and critically reviewed drafts of the manuscript. K.M.L.L. and F.M.S. interpreted the data and critically reviewed drafts of the manuscript. B.M.S. contributed to data collection, data processing, and critically reviewed drafts of the manuscript. P.C.D., C.J.H.v.d.K., N.C.S., M.T.S., and S.J.S.S. developed The Maastricht Study concept and protocol, and critically reviewed drafts of the manuscript. All authors gave final approval of the version of the manuscript to be published. R.J.H.M. and C.D.A.S. are the guarantors of this work.

Sources of funding: The Maastricht Study was supported by the European Regional Development Fund via OP-Zuid, the Province of Limburg, and the Dutch Ministry of Economic Affairs (grant 310.041); Stichting De Weijerhorst (Maastricht, the Netherlands); the Pearl String Initiative Diabetes (Amsterdam, the Netherlands); the Cardiovascular Center (CVC, Maastricht, the Netherlands); CARIM School for Cardiovascular Diseases (Maastricht, the Netherlands); CAPHRI School for Public Health and Primary Care (Maastricht, the Netherlands); NUTRIM School for Nutrition and Translational Research in Metabolism (Maastricht, the Netherlands); Stichting Annadal (Maastricht, the Netherlands); Health Foundation Limburg (Maastricht, the Netherlands); and by unrestricted grants from Janssen-Cilag B.V. (Tilburg, the Netherlands), Novo Nordisk Farma B.V. (Alphen aan den Rijn, the Netherlands), and Sanofi-Aventis Netherlands B.V. (Gouda, the Netherlands). In addition, this study was supported by an unrestricted grant from Fresenius Medical Care (Bad Homburg, Germany).

\section{Conflicts of interest}

There are no conflicts of interest.

\section{REFERENCES}

1. Fox CS, Matsushita K, Woodward M, Bilo HJ, Chalmers J, Heerspink HJ, et al. Associations of kidney disease measures with mortality and endstage renal disease in individuals with and without diabetes: a metaanalysis. Lancet 2012; 380:1662-1673.

2. Spijkerman AM, Gall MA, Tarnow L, Twisk JW, Lauritzen E, LundAndersen H, et al. Endothelial dysfunction and low-grade inflammation and the progression of retinopathy in Type 2 diabetes. Diabet Med 2007; 24:969-976.

3. Ingelsson E, Sundstrom J, Lind L, Riserus U, Larsson A, Basu S, et al. Low-grade albuminuria and the incidence of heart failure in a community-based cohort of elderly men. Eur Heart J 2007; 28:1739-1745.

4. Barzilay JI, Gao P, O'Donnell M, Mann JF, Anderson C, Fagard R, et al. Albuminuria and decline in cognitive function: The ONTARGET/ TRANSCEND studies. Arch Intern Med 2011; 171:142-150.

5. Martens RJ, Kooman JP, Stehouwer CD, Dagnelie PC, van der Kallen CJ, Kroon AA, et al. Albuminuria is associated with a higher prevalence of depression in a population-based cohort study: the Maastricht Study. Nephrol Dial Transplant 2016; [Epub ahead of print].

6. Stehouwer CD, Smulders YM. Microalbuminuria and risk for cardiovascular disease: analysis of potential mechanisms. J Am Soc Nephrol 2006; 17:2106-2111.

7. Knopman DS. Invited commentary: albuminuria and microvascular disease of the brain-a shared pathophysiology. Am J Epidemiol 2010; 171:287-289.
8. Ter Maaten JM, Damman K, Verhaar MC, Paulus WJ, Duncker DJ, Cheng $\mathrm{C}$, et al. Connecting heart failure with preserved ejection fraction and renal dysfunction: the role of endothelial dysfunction and inflammation. Eur J Heart Fail 2016; 18:588-598.

9. Stehouwer CD, Henry RM, Dekker JM, Nijpels G, Heine RJ, Bouter LM. Microalbuminuria is associated with impaired brachial artery, flowmediated vasodilation in elderly individuals without and with diabetes: further evidence for a link between microalbuminuria and endothelial dysfunction-the Hoorn Study. Kidney Int Suppl (92):2004;S42-S44.

10. Zenere BM, Arcaro G, Saggiani F, Rossi L, Muggeo M, Lechi A. Noninvasive detection of functional alterations of the arterial wall in IDDM patients with and without microalbuminuria. Diabetes Care 1995; 18:975-982.

11. Cosson E, Pham I, Valensi P, Paries J, Attali JR, Nitenberg A. Impaired coronary endothelium-dependent vasodilation is associated with microalbuminuria in patients with type 2 diabetes and angiographically normal coronary arteries. Diabetes Care 2006; 29:107-112.

12. Stehouwer CD, Nauta JJ, Zeldenrust GC, Hackeng WH, Donker AJ, den Ottolander GJ. Urinary albumin excretion, cardiovascular disease, and endothelial dysfunction in noninsulin-dependent diabetes mellitus. Lancet 1992; 340:319-323.

13. Clausen P, Feldt-Rasmussen B, Jensen G, Jensen JS. Endothelial haemostatic factors are associated with progression of urinary albumin excretion in clinically healthy subjects: a 4-year prospective study. Clin Sci (Lond) 1999; 97:37-43.

14. Persson F, Rossing P, Hovind P, Stehouwer CD, Schalkwijk CG, Tarnow L, et al. Endothelial dysfunction and inflammation predict development of diabetic nephropathy in the Irbesartan in Patients with Type 2 Diabetes and Microalbuminuria (IRMA 2) study. Scand J Clin Lab Invest 2008; 68:731-738.

15. Feldt-Rasmussen B. Increased transcapillary escape rate of albumin in type 1 (insulin-dependent) diabetic patients with microalbuminuria. Diabetologia 1986; 29:282-286.

16. Nannipieri M, Rizzo L, Rapuano A, Pilo A, Penno G, Navalesi R. Increased transcapillary escape rate of albumin in microalbuminuric type II diabetic patients. Diabetes Care 1995; 18:1-9.

17. Guglielmi MD, Pierdomenico SD, Salvatore L, Romano F, Tascione E, Pupillo M, et al. Impaired left ventricular diastolic function and vascular postischemic vasodilation associated with microalbuminuria in IDDM patients. Diabetes Care 1995; 18:353-360.

18. Schmiedel O, Schroeter ML, Harvey JN. Microalbuminuria in Type 2 diabetes indicates impaired microvascular vasomotion and perfusion. Am J Physiol Heart Circ Physiol 2007; 293:H3424-H3431.

19. Houben AJ, Schaper NC, Slaaf DW, Tangelder GJ, Nieuwenhuijzen Kruseman AC. Skin blood cell flux in insulin-dependent diabetic subjects in relation to retinopathy or incipient nephropathy. Eur J Clin Invest 1992; 22:67-72.

20. Nakagawa T, Sato W, Glushakova O, Heinig M, Clarke T, CampbellThompson M, et al. Diabetic endothelial nitric oxide synthase knockout mice develop advanced diabetic nephropathy. J Am Soc Nephrol 2007; 18:539-550.

21. Zhao HJ, Wang S, Cheng $\mathrm{H}$, Zhang MZ, Takahashi $\mathrm{T}$, Fogo AB, et al. Endothelial nitric oxide synthase deficiency produces accelerated nephropathy in diabetic mice. J Am Soc Nephrol 2006; 17:2664-2669.

22. Lim M, Sasongko MB, Ikram MK, Lamoureux E, Wang JJ, Wong TY, et al. Systemic associations of dynamic retinal vessel analysis: a review of current literature. Microcirculation 2013; 20:257-268.

23. Roustit M, Cracowski JL. Assessment of endothelial and neurovascular function in human skin microcirculation. Trends Pharmacol Sci 2013; 34:373-384.

24. Nguyen TT, Kreis AJ, Kawasaki R, Wang JJ, Seifert BU, Vilser W, et al. Reproducibility of the retinal vascular response to flicker light in Asians. Curr Eye Res 2009; 34:1082-1088.

25. Agarwal SC, Allen J, Murray A, Purcell IF. Comparative reproducibility of dermal microvascular blood flow changes in response to acetylcholine iontophoresis, hyperthermia and reactive hyperaemia. Physiol Meas 2010; 31:1-11.

26. Schram MT, Sep SJ, van der Kallen CJ, Dagnelie PC, Koster A, Schaper $\mathrm{N}$, et al. The Maastricht Study: an extensive phenotyping study on determinants of type 2 diabetes, its complications and its comorbidities. Eur J Epidemiol 2014; 29:439-451.

27. Sorensen BM, Houben AJ, Berendschot TT, Schouten JS, Kroon AA, van der Kallen CJ, et al. Prediabetes and type 2 diabetes are associated with generalized microvascular dysfunction: the Maastricht Study. Circulation 201613; 4:1339-1352. 
28. Inker LA, Schmid CH, Tighiouart H, Eckfeldt JH, Feldman HI, Greene T, et al. Estimating glomerular filtration rate from serum creatinine and cystatin C.CKD-EPI Investigators $N$ Engl J Med 2012; 367:20-29.

29. Nelson RG, Bennett PH, Beck GJ, Tan M, Knowler WC, Mitch WE, et al. Development and progression of renal disease in Pima Indians with noninsulin-dependent diabetes mellitus. Diabetic Renal Disease Study Group. N Engl J Med 1996; 335:1636-1642.

30. Melsom T, Mathisen UD, Ingebretsen OC, Jenssen TG, Njolstad I, Solbu $\mathrm{MD}$, et al. Impaired fasting glucose is associated with renal hyperfiltration in the general population. Diabetes Care 2011; 34:1546-1551.

31. Siddiqi FS, Advani A. Endothelial-podocyte crosstalk: the missing link between endothelial dysfunction and albuminuria in diabetes. Diabetes 2013; 62:3647-3655.

32. Taddei S, Virdis A, Mattei P, Ghiadoni L, Sudano I, Arrighi P, et al. Lack of correlation between microalbuminuria and endothelial function in essential hypertensive patients. J Hypertens 1995; 13:1003-1008.

33. Arcos MI, Fujihara CK, Sesso A, de Almeida Prado EB, de Almeida Prado MJ, de Nucci G, et al. Mechanisms of albuminuria in the chronic nitric oxide inhibition model. Am J Physiol Renal Physiol 2000; 279:F1060-F1066.

34. Yuen DA, Stead BE, Zhang Y, White KE, Kabir MG, Thai K, et al. eNOS deficiency predisposes podocytes to injury in diabetes. J Am Soc Nephrol 2012; 23:1810-1823.

35. Carlstrom M, Wilcox CS, Arendshorst WJ. Renal autoregulation in health and disease. Physiol Rev 2015; 95:405-511.

36. Houben A, Martens RJH, Stehouwer CDA. Assessing Microvascular Function in Humans from a Chronic Disease Perspective. J Am Soc Nephrol 2017; 28:3461-3472.

\section{Reviewers' Summary Evaluations}

\section{Referee 1}

A major strength of this paper deals with the demonstration of an independent correlation between albuminuria and microvascular endothelial dysfunction, particularly evident in type 2 diabetes patients, a population with a number of potential confounders. Weaknesses are the cross-sectional design, which limits the causality of the associations, and the vascular district explored. Indeed, although the skin microcirculation is a mirror of the systemic microcirculation, the autocrine/paracrine property of the endothelium does not allow considering a vascular district surrogate of another.
37. Nakagawa T. Uncoupling of the VEGF-endothelial nitric oxide axis in diabetic nephropathy: an explanation for the paradoxical effects of VEGF in renal disease. Am J Physiol Renal Physiol 2007; 292: F1665-F1672.

38. Foster RR, Armstrong L, Baker S, Wong DW, Wylie EC, Ramnath R, et al. Glycosaminoglycan regulation by VEGFA and VEGFC of the glomerular microvascular endothelial cell glycocalyx in vitro. Am J Pathol 2013; 183:604-616.

39. Smulders YM, Jager A, Gerritsen J, Dekker JM, Nijpels G, Heine RJ, et al. Cardiovascular autonomic function is associated with (micro-)albuminuria in elderly Caucasian sujects with impaired glucose tolerance or type 2 diabetes: the Hoorn Study. Diabetes Care 2000; 23:1369-1374.

40. Patton N, Aslam T, Macgillivray T, Pattie A, Deary IJ, Dhillon B. Retinal vascular image analysis as a potential screening tool for cerebrovascular disease: a rationale based on homology between cerebral and retinal microvasculatures. J Anat 2005; 206:319-348.

41. Holowatz LA, Thompson-Torgerson CS, Kenney WL. The human cutaneous circulation as a model of generalized microvascular function. J Appl Physiol (1985) 2008; 105:370-372.

42. IJzerman RG, de Jongh RT, Serne EH. Commentary on viewpoint: the human cutaneous circulation as a model of generalized microvascular function. J Appl Physiol (1985) 2008; 105:378.

43. Schisterman EF, Cole SR, Platt RW. Overadjustment bias and unnecessary adjustment in epidemiologic studies. Epidemiology 2009; 20: 488-495.

44. Quyyumi AA, Patel RS. Endothelial dysfunction and hypertension: cause or effect? Hypertension 2010; 55:1092-1094.

\section{Referee 2}

Microalbuminuria is considered not only a marker of renal disease but also of generalized microvascular endothelial function. For the latter there is, however, only limited direct evidence available. In this paper microvascular endothelial function has been assessed and relationships with micoralbuminuria have been explored. The paper not only shows that the proposed concept can be confirmed in cross-sectional data but also reminds us that our methods for assessment of both microalbuminuria and endothelial function remain suboptimal. 